\title{
Road usability for wheelchair users at an international tourist city Kamakura clarified by data collected by "Rin-Reco" (drive recorder exclusively developed for bicycle)
}

\author{
Sadao HORINO (horino@kanagawa-u.ac.jp) ${ }^{1}$, Kazutaka KOGI ${ }^{2}$ \\ ${ }^{1}$ Kanagawa University, Research Institute for Engineering \\ ${ }^{2}$ The Ohara Memorial Institute for Science of Labour
}

\begin{abstract}
Practical methods to utilize a driving event video recorder ("drive recorder" in short) recording the driving situation of a car, the bicycle by camera is becoming widespread. This method include a proactive utility for the preclusive assessment of road infrastructure environment as well as reactive utility for traffic accident investigation. It attracts much attention, since such a record can be applied to user-friendly citizen-based town planning as well.
\end{abstract}

ABSTRACT

Keywords: "Rin-Reco", user-friendly citizen-based town planning, group discussion

\section{Method}

The authors repeated a continuous effort for last 14 years since 2003 to make International Tourist City Kamakura barrier-free by improving road design, access to stores, public facilities such as railway stations, the restrooms at stations, shrines and temples from wheel-chair user's point of view in the ergonomic universal design context. We still repeat further effort to utilize a moving picture recorded by "drive recorder" of traffic movement situation for barrier-free improvement in the significant proposals to citizen-based town planning.
We utilized the recorded moving picture collected by a handy continuously recording type "drive recorder" called "Rin-Reco" which was exclusively developed for bicycle for barrier-free improvement suggestion to user-friendly citizen-based town planning. This handy type"Rin-Reco" was installed on the armrest of the wheelchair, was powered by two batteries of size AAA and recorded the forward scene by camera with view angle 63 degree, a sound, the speed and the GPS space information simultaneously during the traffic move by feet self-driven and electric wheelchair (Figure 1).

In occasion of The Kamakura Barrier-Free Symposium in 2015 and 2016, we projected a recorded moving picture of the wheelchair traffic on the general road (1),(2) and the sightseeing road (3) in Kamakura, and the participants are invited to an open and free discussion to introduce their comments in small groups, and finally the result of group discussion was summarized altogether (Figure 2).

Through such bottom-up procedure, the whole participants finally got agreed proposals to improve the roads in Kamakura. This procedure includes the following 
steps; after the whole participants watched the moving picture on screen for about 30 minutes, they had group discussion in small groups and summarized good points as well as points to be improved of the road in Kamakura in each groups and through exchange of discussion results, they conducted a plenary session, and tried to agree an integrated positive action based suggestions for the next citizen-based town planning.

\section{Results}

\section{$<$ Items clarified by a moving picture analysis recorded with video camera mounted on the wheelchair >}

It revealed that the forward picture record during the traffic with a wheelchair could provide a plenty of road environmental information such as the road width, the traffic division mark, the road-surface condition and the surface inclination angle, the degree of curve change, the road surface mark, the indication of a manhole $\&$ the fire hydrant, maintenance condition of the gutter cover, sidewalk step and hollow, the obstacles such as telephone poles, the signboard and planting, the movement of passing traffic, and this information contributed to discussion on the moving safety and obstruction factors of wheelchair traffic.

In addition, further information were provided according to a traffic condition, in regard with the situation facing difficulty in wheelchair movement, the interrelated situation with the passing pedestrians, near-accident situation such as contact with a passing car. It was remarkable to learn a real viewpoint of on-site wheelchair traffic, since the lens axis of the wheelchair mounted "Rin-Reco" camera was low with $0.5 \mathrm{~m}$ above the ground, and the speed was $3-5 \mathrm{~km} / \mathrm{h}$. A moving picture record was greatly helpful to realize a deadlock while congestion, a feeling of stress by the blockage.

$<$ Good points / points to be improved of the road at Kamakura from wheelchair users view point $>$

Being inspired by such a moving picture record, the discussion among the symposium participants offered them a good learning opportunity, and the method to discuss points to be improved after discussing good points was helpful very much to propose concrete suggestions. The major results of the group discussion in 2015 and 2016 were shown in a form of comparison (Table 1).

The result of group discussion after sharing a moving picture record by "Rin-Reco" mounted on wheelchair showed that more multifaceted remarks were pointed out rather than merely picking up factors of safety distraction.

The most popular good points were concentrated in the wide spread of ensuring sufficient traffic road (Figure 3), the relocation of obstacles (Figure 4), mutual concessions, cooperation between the passing tourists (Figure 5).

We classified the degree of barrier-free of a telephone pole installed in the road border with $\mathrm{A}, \mathrm{B}, \mathrm{C}$ rank and analyzed their distribution from wheelchair traffic point of view. A: very kind to wheelchair traffic, no interference by locating in the residential land. B: kind to wheelchair traffic: located inside the gutter-cover, at the border of the sidewalk and the road, at the corner cut-off. C: interfere with wheelchair traffic: located at beyond the road boundary. As for 31, 58, 47 telephone poles installed in the route (1),(2) and (3), the most of them were kind to wheelchair traffic, namely 19, 29, $49 \%$ of them respectively were classified with A rank (Figure 3), 42, 71, 45\% of them respectively were classified with B rank, and remarkably $90 \%$ of telephone poles located at the left side sidewalk of the route(3) from the Hase Station of the Enoshima Railway to the Great Buddha were A rank.

We could point out the importance of identifying the maintenance situation of the road and its neighboring conducted by stores, the passing tourists as well as by the city authority. We could learn that visible confirmation of the collaborative effort under such local condition surely ensured one basic point of the user friendly citizen-based town planning.

As for the current points to be improved with both year, many comments were pointed out in regard with securing safe road by traffic separation between pedestrians and cars and removal of sidewalk steps. In addition, the consideration about blockages such as a telephone pole and the planting was common in both year, too. And it was frequently concerned during the 
discussion with a proposal for the road safety culture construction with the emphasis on "the barrier-free of the heart".

\section{Conclusion}

We could confirm various factors kind to wheelchair traffic through open discussion on "Rin-Reco" moving picture record which were achieved by creative ideas and much efforts by civil administration, inhabitants and the passing tourists, while the road in Kamakura as for the International Tourist City is narrow, and lot of difficult issues are existing toward making the road infrastructure more barrier-free.

We could admit that moving picture by handy type drive recorder "Rin-Reco" mounted on wheelchair and the relevant discussion were extremely significant in directing and suggesting an improvement depended on such efforts.

We could confirm the effectiveness of group works focused on the real moving picture acquired from on-site wheelchair traffic to indicate the direction of collaborative effort in the future. We expect further development in the application of moving picture of wheelchair traffic in combined with the spread of drive recorder utilization to road traffic safety.

\section{Acknowledgements}

This work was conducted by cooperation of Kamakura barrier free study group. 